\title{
Impedance-Based Immobilized Enzyme Biosensor for Detection of Organophosphates
}

\author{
M.F. Smiechowski ${ }^{1}$, A.R. Ward ${ }^{1}$ \\ ${ }^{1}$ Guild Associates, Inc. 5750 Shier-Rings Rd., Dublin, Ohio, USA \\ msmiechowski@guildassociates.com
}

\begin{abstract}
:
This paper describes the development of an electrochemical impedance-based biosensor for the detection of organophosphates. The biosensor consisted of a carbon paste electrode, modified with an immobilized organophosphate-sensitive enzyme layer. The enzyme was immobilized through crosslinking with glutaraldehyde, polyethyleneimine, and additional cell material. The sensor signal and response to paraoxon substrate was tested versus two different electrode arrangements and over several applied dc potentials. The sensor was capable of measuring $10 \mu \mathrm{M}$ paraoxon in buffer solution. Stability of the sensor stored in air over time was investigated. Results are shown for the biosensor versus a paraoxon substrate operating in $50 \mathrm{mM}$ HEPES buffer at $150 \mathrm{mV}, 0 \mathrm{mV}$, and -150 $\mathrm{mV}$ vs. $\mathrm{Ag} / \mathrm{AgCl}$.
\end{abstract}

Key words: electrochemical impedance, organophosphates, biosensor, immobilized enzyme

\section{Introduction}

Organophosphate (OP) compounds are commonly used as pesticides, insecticides, and as chemical warfare agents. Although OP use as a chemical warfare agent has been restricted by international treaties, OP contamination is still possible as these compounds are widely used in agricultural and domestic pest controls [1-3]. For example, over 13 million $\mathrm{kg}$ of OP pesticides have been used annually in the U.S., representing $40 \%$ of the world market in insecticide use [3]. With this widespread use of toxic compounds there are legitimate concerns about contamination of soil and water systems. Consequently there is a need for simple, rapid, and reliable methods for OP detection and decontamination monitoring.

A number of electrochemical biosensors have been investigated in an attempt to satisfy this need based on either indirect detection through cholinesterase enzyme inhibition, or by direct detection through the use of organophosphate hydrolase $(\mathrm{OPH})$ and organophosphorus acid anhydrolase (OPAA) [4, 5]. Biosensors based on these enzymes offer the advantages of direct detection of a wide range of OPs, and simple, direct, and rapid measurements without the need to reactivate/regenerate the enzyme on the electrode.

Electrochemical impedance spectroscopy (EIS) has a high potential for characterizing the biocatalytic processes at enzyme modified electrode surfaces [6, 7]. The measurements can be performed at low $\mathrm{AC}$ frequencies at a fixed potential, to allow for the monitoring of the reactions taking place at the electrode surface. At the same time, high AC frequency measurements can provide information about changes in the bulk solution.

\section{Experimental}

OP biosensors were constructed using equal amounts of wild type OPH and OPAA enzymes immobilized with glutaraldehyde, polyethyleneimine, and lysed cell material from A. niger. The immobilized material was ground to a fine powder. Electrodes were constructed using a $3 \mathrm{~mm}$ diameter carbon paste electrode probe. The first variation replaced about $1 \mathrm{~mm}$ of the carbon paste surface with immobilized enzyme powder. The second variation blended the immobilized enzyme with the graphite at a 1:1: ratio, prior to the addition of paraffin oil. This material was then used to construct a working electrode.

Impedance tests were performed using a Parastat 2263 electrochemical workstation (Princeton Applied Research, Oak Ridge, TN). EIS spectra were measured across ac frequencies of $1 \mathrm{MHz}$ to $50 \mathrm{mHz}$, with an amplitude of $10 \mathrm{mV}$. Prior testing with platinum and glassy carbon working electrodes was used to determine the dc potential of $-150 \mathrm{mV}$ vs. $\mathrm{Ag} / \mathrm{AgCl}$ for these experiments testing. Kinetics of any enzyme/paraoxon reactions due to the presence of the enzyme in the test solution were independently monitored through absorbance measurements at $410 \mathrm{~nm}$. 


\section{Results and Discussion}

Initial tests were performed using a bare carbon paste electrode versus concentrations of paraoxon from 0 to $100 \mu \mathrm{M}$, Fig 1. Although not illustrated in Fig, 1. the impedance response to paraoxon showed two distinct relaxations, one at a high frequency $(\sim 100 \mathrm{kHz})$ and a second at a frequency closer to the end point of the tested range $(\sim 50 \mathrm{mHz})$.

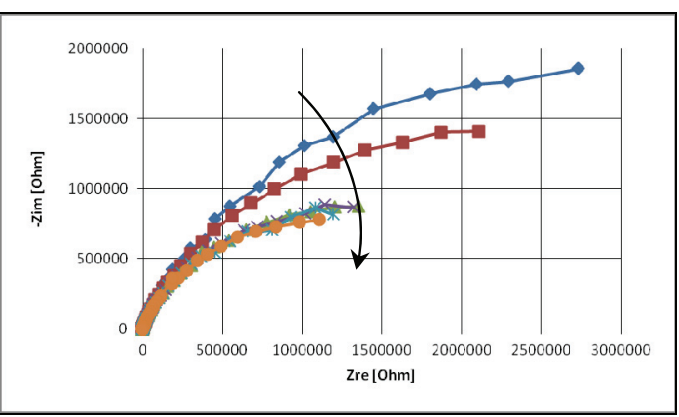

Fig. 1: Nyquist plot of typical EIS data from a bare carbon paste electrode at $-150 \mathrm{mV} v \mathrm{~s}$. $\mathrm{Ag} / \mathrm{AgCl}$. The arrow indicates increase in paraoxon concentration.

The impedance response of the carbon paste electrode was similar at all three potentials it was measured. An initial decrease in measured impedance was seen as the concentration increased from 0 to $20 \mu \mathrm{M}$, as the concentration of paraoxon was further increased, the measured impedance continued to decrease, but at a slower rate, Tab. 1.

Tab. 1: EIS data measured at $100 \mathrm{~Hz}$ for a bare carbon paste working electrode vs. paraoxon concentration and applied dc potential.

\begin{tabular}{|c|c|c|c|}
\hline$|Z|[k O h m]$ & $-150 \mathrm{mV}$ & $0 \mathrm{mV}$ & $150 \mathrm{mV}$ \\
\hline $0 \mu \mathrm{M}$ & 13.4 & 15.4 & 13.1 \\
\hline $10 \mu \mathrm{M}$ & 10.7 & 11.0 & 10.0 \\
\hline $20 \mu \mathrm{M}$ & 7.0 & 6.9 & 6.5 \\
\hline $50 \mu \mathrm{M}$ & 6.3 & 6.3 & 6.1 \\
\hline $75 \mu \mathrm{M}$ & 6.0 & 5.9 & 5.7 \\
\hline $100 \mu \mathrm{M}$ & 5.4 & 5.3 & 5.1 \\
\hline
\end{tabular}

The pattern seen at $100 \mathrm{~Hz}$ is preserved at lower frequencies; however, at frequencies above $10 \mathrm{kHz}$, the decrease in impedance with increasing paraoxon concentration becomes less significant.

Testing with immobilized enzyme examined two methods of electrode construction in order to explore any advantages in functionality in analysis of paraoxon solutions. The two construction methods (on surface and blended) were compared to an unaltered carbon paste electrode where the immobilized enzyme was added to the paraoxon test solution, Fig 2. A similar amount of immobilized enzyme was used in each test case.

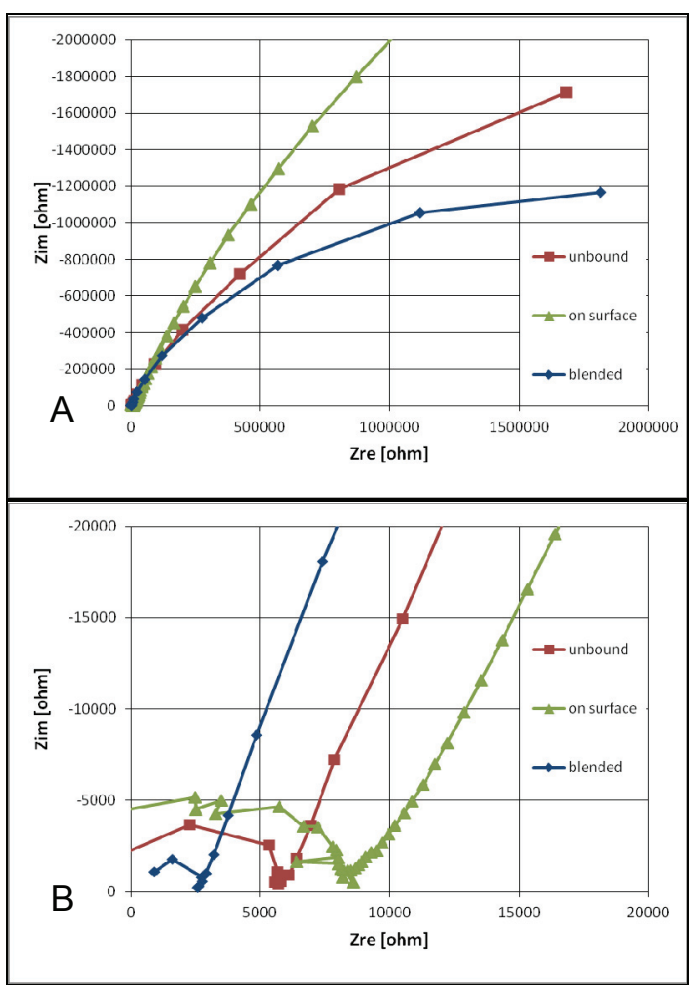

Fig. 2: Impedance measurements of a $50 \mathrm{mM}$ paraoxon buffer solution at $-150 \mathrm{mV}$ vs. $\mathrm{Ag} / \mathrm{AgCl}$ of using a carbon paste working electrode with variations of immobilized enzyme. B) shows an expanded portion of the lower left corner of $A$ ).

The immobilized enzyme layered on the carbon paste electrode surface produced a significantly different result from the other two tests. Overall the measured impedance for this system is significantly larger than the other two. Also there is the possible presence of a third relaxation near $1 \mathrm{kHz}$ for this arrangement attributed to the layer of immobilized enzyme on the surface of the electrode. The electrode with the blended immobilized enzyme does not have this feature, but compared to the carbon paste electrode shows an overall reduced impedance. This could be due an opening up the carbon paste electrode structure and to the immobilized enzyme both binding with the paraoxon and producing electroactive byproducts inside of the electrode.

The addition of immobilized enzyme to the electrodes for these tests can convert the paraoxon to p-nitrophenol over time. This conversion was not significant for either of the tests where the enzyme was part of the electrode; however, it was noticeable for the 
test where the enzyme was just added to the paraoxon solution. While the EIS sweep was rapid ( $<10$ minutes), the conversion of paraoxon likely effected the test measurements. Absorbance measurements of the test solution confirmed that roughly one third of the paraoxon was converted to $p$-nitrophenol during that particular test.

Further testing of the carbon paste electrode design with an immobilized enzyme surface layer was performed to determine the impedance response to a wider range of paraoxon concentrations, Tab. 2.

Tab. 2: EIS data measured at $100 \mathrm{~Hz}$ for a carbon paste working electrode with a $1 \mathrm{~mm}$ surface layer of immobilized enzyme vs. paraoxon concentration and applied dc potential.

\begin{tabular}{|c|c|c|c|}
\hline$|Z|[k O h m]$ & $-150 \mathrm{mV}$ & $0 \mathrm{mV}$ & $150 \mathrm{mV}$ \\
\hline $0 \mu \mathrm{M}$ & 32.5 & 36.4 & 31.0 \\
\hline $10 \mu \mathrm{M}$ & 28.7 & 29.2 & 30.4 \\
\hline $20 \mu \mathrm{M}$ & 27.4 & 27.7 & 28.9 \\
\hline $50 \mu \mathrm{M}$ & 25.6 & 25.8 & 27.0 \\
\hline $75 \mu \mathrm{M}$ & 24.6 & 24.7 & 25.9 \\
\hline $100 \mu \mathrm{M}$ & 23.9 & 24.0 & 25.3 \\
\hline
\end{tabular}

The results from these tests are similar to the bare carbon paste electrode tests shown in Table 1. In the case of the immobilized enzyme, the change in impedance with respect to concentration is closer to linear throughout the tested concentration range and at each potential, unlike the disjunction seen with the bare carbon paste electrode.

Further analysis of the immobilized enzyme electrode involved determining its stability over time while stored in air at room temperature. Theoretically, this method of immobilization should provide the enzymes with an increased resistance to the destabilizing effects of heat and dehydration. Impedance measurements were performed using a carbon paste working electrode with an immobilized enzyme layer on its surface. Between measurements the electrode was stored in open air at room temperature.

Results from this testing show, Fig. 3 and Tab. 3 , that the initial exposure to air between tests increased the measured impedance of the sensor. The most significant changes were noted in the low frequency $(<1 \mathrm{kHz})$ portions of the EIS data, Fig. 3. The real and imaginary components of the impedance in increased each day till the third day of testing, where they appeared to stabilize. While, there was little change in the shape of the data at frequencies over $1 \mathrm{kHz}$ over the course of this testing.

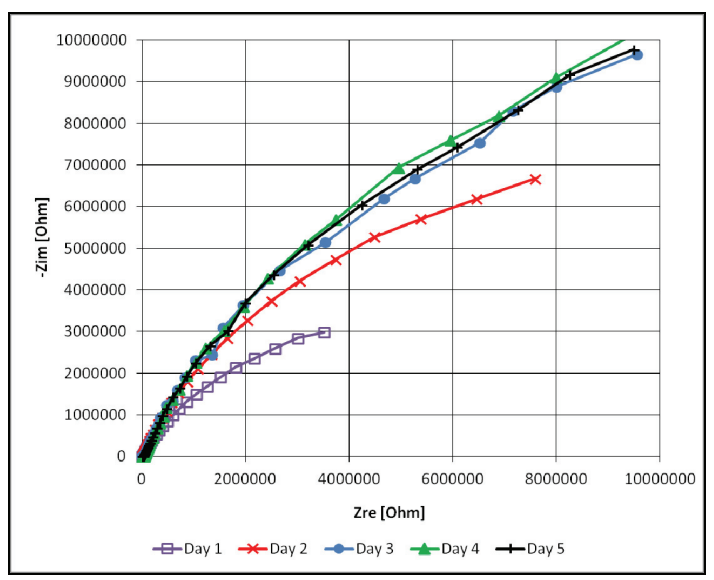

Fig. 3: Impedance measurements of a carbon paste/immobilized enzyme electrode stored in open air at room temperature. Samples were tested against at $50 \mathrm{mM}$ paraoxon buffer solution at -150 $m V$ vs. $\mathrm{Ag} / \mathrm{AgCl}$.

Testing was also performed versus a number of paraoxon concentrations over the five days. Tab. 3 lists the measured value for $|Z|$ at each of the paraoxon concentrations tested. Although the measured impedance increases over time, the linear relationship between to concentration remains. Over time the slope of the linear relationship increases, the slope on day 2 is twice that of day 1 , and the slope of the curve on day 3 is 3 times that of day 1 . The increased slope would suggest an increased sensitivity to the concentration of paraoxon with time, suggesting that a further stabilization of the immobilized material may be taking place as the electrode dries.

Tab. 3: EIS data measured at $100 \mathrm{~Hz}$ for a carbon paste working electrode with a $1 \mathrm{~mm}$ surface layer of immobilized enzyme vs. $50 \mu \mathrm{M}$ paraoxon at a dc potential of $-150 \mathrm{mV}$ over time stored in air.

\begin{tabular}{|c|c|c|c|c|c|}
\hline $\begin{array}{c}|\mathrm{Z}| \\
{[\mathrm{kOhm}]}\end{array}$ & $\begin{array}{c}\text { Day } \\
1\end{array}$ & $\begin{array}{c}\text { Day } \\
2\end{array}$ & $\begin{array}{c}\text { Day } \\
3\end{array}$ & $\begin{array}{c}\text { Day } \\
4\end{array}$ & $\begin{array}{c}\text { Day } \\
5\end{array}$ \\
\hline $0 \mu \mathrm{M}$ & 32.5 & 42.6 & 66.8 & 58.1 & 62.3 \\
\hline $10 \mu \mathrm{M}$ & 28.7 & 38.7 & 62.1 & 53.7 & 56.5 \\
\hline $20 \mu \mathrm{M}$ & 27.4 & 35.2 & 57.6 & 48.1 & 52.2 \\
\hline $50 \mu \mathrm{M}$ & 25.6 & 31.8 & 53.1 & 44.2 & 48.4 \\
\hline $75 \mu \mathrm{M}$ & 24.6 & 28.4 & 49.1 & 40.1 & 42.5 \\
\hline $100 \mu \mathrm{M}$ & 23.9 & 26.8 & 45.8 & 37.8 & 40.1 \\
\hline
\end{tabular}

\section{Conclusions}

We have investigated the application of immobilized enzyme for an EIS-based biosensor for the detection of an 
organophosphate nerve agent. The addition of immobilized OPH/OPAA to the electrode showed increased sensitivity to the presence of paraoxon in solution, while maintaining acceptable stability over time. The stability of the immobilized enzyme combined with the ease of assembly of the electrode can provide a route towards a sensor device that is easier to store and employ as needed.

\section{Acknowledgements}

The work was funded by Guild Associates, Inc.

\section{References}

[1] S. Chauhan, S. Chauhan, R. D'Cruz, S. Faruqi, K.K. Singh, S. Varma, M. Singh, V. Karthik, Environ. Toxicol. Pharmacol. 26, 113-122 (2008); doi:10.1016/j.etap.2008.03.003

[2] M.R. Bonner, J. Coble, A. Blair, L.E. Beane Freeman, J.A. Hoppin, Dale P. Sandler, M.C.R. Alavanja, Am. J. Epidemiol. 166 (9): 1023-1034 (2007); doi: 10.1093/aje/kwm182

[3] A. Grube, D. Donaldson, T. Kiely, L. Wu, Pesticides industry sales and usage: 2006 and 2007 market estimates. Washington, DC: Environmental Protection Agency; (2011).

[4] A. Mulchandani, W. Chen, P. Mulchandani, J. Wang, K.R. Rogers, Biosensors and Bioelectronics, 16, 225-230 (2001); doi:10.1016/S0956-5663(01)00126-9.

[5] A.L. Simonian, J.K. Grimsley, A.W. Flounders, J.S. Schoeniger, Tu-Chen Cheng, J.J. DeFrank, J.R. Wild, Anal. Chim. Acta., 442, 15-23 (2001); doi: 10.1016/S0003-2670(01)01131-X

[6] M. Zayats, E. Katz, I. Willner, J. Am. Chem. Soc., 124 (49), 14724-14735 (2002); doi: 10.1021/ja027919y

[7] E.J. Calvo, R. Etchenique, C. Danilowicz, L. Diaz, Anal. Chem. 68, 4186 (1996); doi: 10.1021/ac960170n 